\title{
ON RELATIVE NORMAL COMPLEMENTS IN FINITE GROUPS. III
}

\author{
HENRY S. LEONARD, JR.
}

\begin{abstract}
Let $G$ be a finite group, let $H \leq G$, and let $\pi$ be the set of prime divisors of $|H|$. Assume that whenever two elements of $H$ are $G$-conjugate then they are $H$-conjugate. Assume that for all $h \in H^{\#},\left(C_{G}(h): C_{H}(h)\right)$ is a $\pi^{\prime}-$ number. We prove that $H$ is a $\pi$-Hall subgroup and that there exists a normal complement $G_{0}=G-\left(H^{\#}\right)^{G, \pi}$. An example shows that the generalization to relative normal complements is not true.
\end{abstract}

This paper continues the studies made in two earlier papers [2, 3]. Our object here is to prove the following theorems.

THEOREM 1. Let $G$ be a finite group, let $H \leq G$, and let $\pi$ be the set of prime divisors of $|H|$. Assume the following conditions:

(A) Whenever two elements of $H$ are $G$-conjugate then they are $H$-conjugate.

$\left(\mathrm{B}_{1}\right)$ For every nonidentity $\pi$-element $h \in H,\left(C_{G}(h): C_{H}(h)\right)$ is a $\pi^{\prime}$-number.

Then $H$ is a $\pi$-Hall subgroup of $G$, and there exists a unique normal complement $G_{0}=G-\left(H^{\#}\right)^{G, \pi}$ of $H$ in $G$.

Conversely, given a $\pi$-Hall subgroup $H$ of $G$ and a normal complement $G_{0}$ of $H$, conditions $(\mathrm{A})$ and $\left(\mathrm{B}_{1}\right)$ must hold.

Here we mean that $G_{0} \Delta G, G_{0} H=G$, and $G_{0} \cap H=\{1\}$. The statement that $H$ is a $\pi$-Hall subgroup means that $|H|$ is divisible only by primes in $\pi$ and that no prime in $\pi$ divides $(G: H)$. Each element $x$ of $G$ has a unique decomposition, $x=x_{\pi} x_{\pi^{\prime}}=x_{\pi^{\prime}} x_{\pi}$, into a $\pi$-element $x_{\pi}$ and a $\pi^{\prime}$-element $x_{\pi^{\prime}}$. Both $x_{\pi}$ and $x_{\pi^{\prime}}$ are powers of $x$. Two elements $x$ and $y$ of $G$ belong to the same $\pi$-section of $G$ if their $\pi$-parts $x_{\pi}$ and $y_{\pi}$ are $G$-conjugate. If $S$ is a subset of $G$ we let $S^{G, \pi}$ denote the union of all $\pi$-sections of $G$ that intersect $S$.

Under stronger assumptions than our conditions $(A)$ and $\left(B_{1}\right), G$. Robinson has proved a theorem asserting the existence of a relative normal complement $[4$, Theorem D]. His theorem is itself a generalization of earlier results. (See his references.)

An example will show that the generalization to relative normal complements of Theorem 1 is not true. Let $G$ be the $p$-group described in [2, §5, Example 1]. Let $H$ and $H_{0}$ be the subgroups described there. According to [2, Proposition 5.1] we have

THEOREM 2. Let $G$ be the p-group referred to, with $H_{0} \Delta H \leq G$ as described. Then:

(i) Whenever two elements $x_{1}$ and $x_{2}$ of $H$ are $G$-conjugate then $x_{1} H_{0}=x_{2} H_{0}$.

Received by the editors November 26, 1984.

1980 Mathematics Subject Classification. Primary 20D20. 
(ii) If $x \in H-H_{0}$ then $C_{G}(x)=H$.

(iii) There is no relative normal complement $G_{0}$ of $H$ over $H_{0}$.

By definition a relative normal complement $G_{0}$ of $H$ over $H_{0}$ would be a normal subgroup $G_{0}$ of $G$ such that $G_{0} H=G$ and $G_{0} \cap H=H_{0}$.

ProOF OF THEOREM 1. Let $P$ be a Sylow $p$-subgroup of $H$ for some $p \in \pi$. Suppose $P$ is not a Sylow $p$-subgroup of $G$. Then some $p$-subgroup $Q$ of $G$ contains $P$ as a proper normal subgroup. Then $Z(Q) \cap P \neq\langle 1\rangle$. Let $k$ be a nonidentity element of $Z(Q) \cap P$. Then $Q \subseteq C_{G}(k)$ but $Q \nsubseteq H$. Thus $p \mid\left(C_{G}(k): C_{H}(k)\right)$, contrary to hypothesis $\left(\mathrm{B}_{1}\right)$.

Thus $P$ is a Sylow $p$-subgroup of $G$, and $H$ is a $\pi$-Hall subgroup of $G$. Therefore $G$ satisfies the hypotheses of a Theorem of Brauer [1, Theorem 3]. (Also see his Remark 1, p. 81.)

The converse part of our Theorem 1 follows directly from Brauer's Theorem 3 and his Remark 1 [1].

\section{REFERENCES}

1. R. Brauer, On quotient groups of finite groups, Math. Z. 83 (1964), 72-84.

2. H. S. Leonard, On relative normal complements in finite groups, Arch. Math. (Basel) 40 (1983), 97-108.

3. __, On relative normal complements in finite groups. II, Proc. Amer. Math. Soc. 88 (1983), 212-214.

4. G. R. Robinson, Blocks, isometries and sets of primes, Proc. London Math. Soc. (to appear).

DEPARTMENT OF MATHEMATICAL SCIENCES, NORTHERN ILlinOIS UNIVERSITY, DEKALB, ILLINOIS 60115 